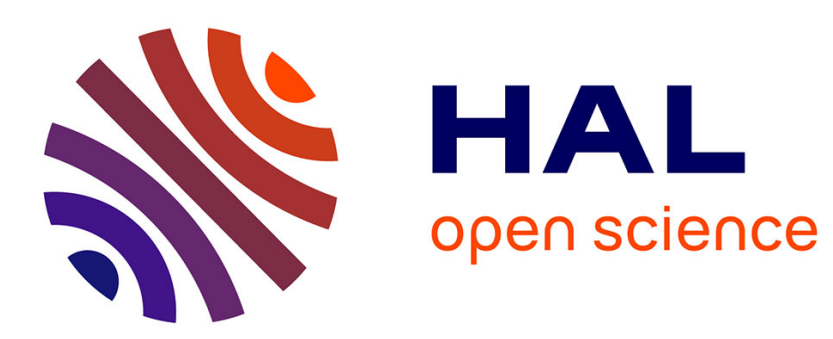

\title{
Réalisation d'une impulsion géante unique dans un laser à rubis
}

\author{
Yves Le Duff, Pierre Peretti, René Dupeyrat
}

\section{To cite this version:}

Yves Le Duff, Pierre Peretti, René Dupeyrat. Réalisation d'une impulsion géante unique dans un laser à rubis. Revue de Physique Appliquée, 1966, 1 (2), pp.117-119. 10.1051/rphysap:0196600102011700 . jpa-00242694

\section{HAL Id: jpa-00242694 https://hal.science/jpa-00242694}

Submitted on 1 Jan 1966

HAL is a multi-disciplinary open access archive for the deposit and dissemination of scientific research documents, whether they are published or not. The documents may come from teaching and research institutions in France or abroad, or from public or private research centers.
L'archive ouverte pluridisciplinaire HAL, est destinée au dépôt et à la diffusion de documents scientifiques de niveau recherche, publiés ou non, émanant des établissements d'enseignement et de recherche français ou étrangers, des laboratoires publics ou privés. 


\title{
RÉALISATION D'UNE IMPULSION GÉANTE UNIQUE DANS UN LASER A RUBIS
}

\author{
Par Yves LE DUfF, Pierre PERETTi et René DUPEyRAT, \\ Laboratoire des Recherches Physiques de la Faculté des Sciences de Paris.
}

\begin{abstract}
Résumé. - Deux méthodes classiques de " déclenchement » des lasers à rubis sont rappelées (miroir tournant, verre non linéaire). Leur association est avantageuse car elle donne avec certitude une seule impulsion dont la largeur est réduite et la hauteur accrue ce qui correspond à un gain de puissance.
\end{abstract}

Abstract. - We discuss two classical methods of switching ruby lasers to give “ a giant pulse " (rotating mirror and non linear glass).

Combinaison of the two methods is advantageous since it gives a single pulse wich is shorter ond of higher amplitude, with a corresponding power gain.

La production du " giant pulse » à l'aide d'un laser à rubis est connue depuis quelques années; les procédés mis en cuvre s'appuient sur un même principe : réduction du facteur de qualité de la cavité pendant un certain temps, puis augmentation brusque afin de libérer l'énergie dans le temps le plus court possible.

Nous avons examiné deux des procédés déjà existants ; ils utilisent :

- un miroir tournant ;

- une substance à transmission non linéaire.

« Déclenchement " par miroir tournant. - Sur un banc d'optique sont disposés, un miroir tournant dont l'axe de rotation est perpendiculaire à l'axe géométrique du rubis, l'enceinte réflectrice contenant le rubis et le flash, un miroir partiellement réflecteur constitué ici par la face de sortie du rubis (fig. 1).
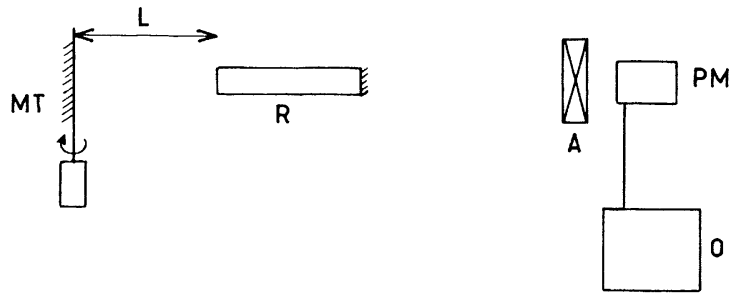

FIG. 1. - Schéma d'un laser à rubis déclenché par miroir tournant.

MT : miroir tournant ; $\mathrm{R}$ : rubis ; A : atténuateur ; $\mathrm{PM}$ : photomultiplicateur ; $\mathrm{O}$ : oscillographe.

Un dispositif électronique convenable retarde de $\Delta T$ de l'ordre de $200 \mu$ s, l'émission laser par rapport à l'amorçage du flash (cette émission n'est en effet possible que pendant un court instant lorsque le miroir tournant occupe une position sensiblement perpendiculaire à l'axe géométrique du rubis).
Dans le laser que nous avons utilisé (cristal de longueur $150 \mathrm{~mm}$, diamètre $8 \mathrm{~mm}$ ) la distance $L$ miroir-rubis est de l'ordre de $10 \mathrm{~cm}$, la vitesse de rotation du miroir contrôlée à l'oscillographe est 29200 tours/minute.

Dans ces conditions, le flux lumineux laser étant reçu sur un récepteur photoélectrique on obtient généralement un oscillogramme présentant plusieurs impulsions ( $f g .2$ ).

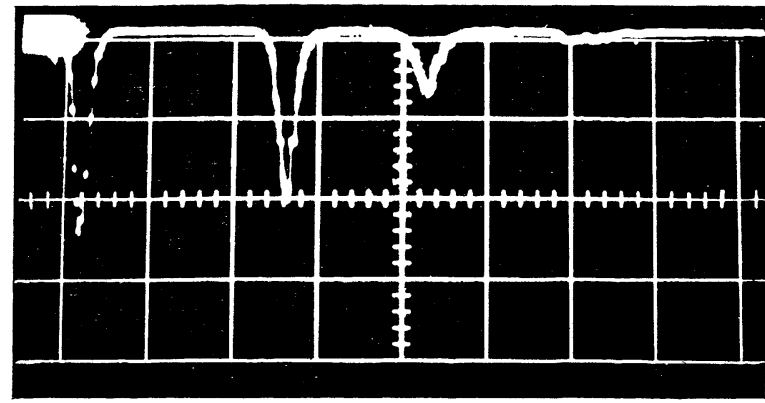

Fig. 2. - Oscillogramme d'un éclair de laser déclenché par miroir tournant.

Sensibilité : $1 \mathrm{~V} / \mathrm{cm}$; balayage : $200 \mathrm{~ns} / \mathrm{cm}$.

Ces impulsions ont en général une largeur à mihauteur de $40 \mathrm{~ns}$. L'intervalle entre deux impulsions étant de l'ordre de quelques centaines de nanosecondes pour une énergie de 2500 joules dans le flash. La puissance transportée par le faisceau pour chaque impulsion est déterminée par la hauteur mesurée sur l'oscillogramme après étalonnage préalable.

$N$. B. : Il est possible en modifiant les paramètres du système (notamment $L$ et $\Delta T$ ) d'obtenir une impulsion unique; mais dans ce cas la puissance n'augmente pas de façon notable.

« Déclenchement » par substance à transmission 
non linéaire. - Certaines substances voient leur transmission augmenter brusquement lorsque l'intensité lumineuse du faisceau incident croît au-delà d'un certain seuil. Si le phénomène s'établit dans un temps assez court (inférieur à $10^{-8} \mathrm{~s}$ pour les phtalocyanines [1]), elles jouent le rôle d'interrupteur optique rapide et peuvent permettre dans certains cas le "déclenchement " de l'émission laser ; il en est ainsi des phtalocyanines et de quelques verres Schott [2].

Notre montage comprend dans l'ordre : un miroir totalement réflecteur fixe, un verre Schott RG 8, le rubis et uı miroir partiellement réflecteur ( $f g .3)$.
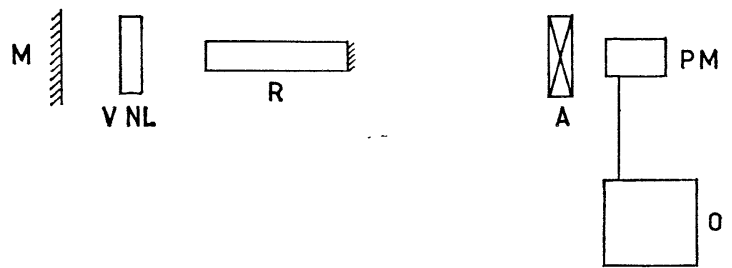

Fig. 3. - Schéma d'un laser à rubis déclenché par verre non linéaire.

$\mathrm{M}$ : miroir ; VNL : verre non linéaire ; $\mathrm{A}$ : atténuateur ; PM : photomultiplicateur ; $\mathrm{O}$ : oscillographe

Pour une énergie de 1600 joules dans le flash, nous obtenons à l'oscillographe deux impulsions principales distantes de plusieurs microsecondes. Une augmentation de l'énergie dans le flash multiplie le nombre d'impulsions sans accroìtre la puissance maximale (fig. 4).

$N$. B. : Ici encore nous pourrions obtenir une seule impulsion en modifiant certains paramètres mais sans gain de puissance si l'on ne modifie pas la longueur du rubis.

"Déclenchement " par association d'un verre à transmission non linéaire et d'un miroir tournant. $\mathrm{Si}$, pour diminuer les pertes, nous réduisons la distance $L$ (miroir tournant - rubis) dans la première méthode, le nombre de "pulses" augmente. L'exis-

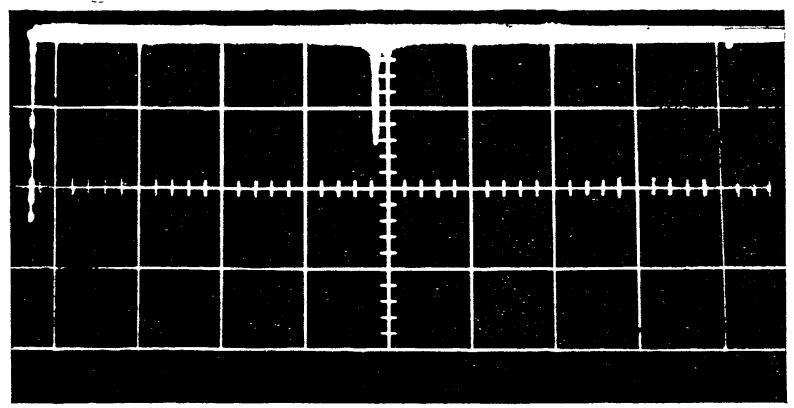

FIG. 4. - Oscillogramme d'un éclair de laser déclenché par verre non linéaire.

Sensibilité : $0,5 \mathrm{~V} / \mathrm{cm}$;balayage : $5 \mu \mathrm{s}$. tence de plusieurs impulsions prouve que la condition d'oscillation est réalisée pour plusieurs orientations du miroir de part et d'autre de la position correspondant au parallélisme du miroir et des faces du rubis.

En introduisant un verre non linéaire entre le miroir tournant et le rubis nous retardons artificiellement le moment où le facteur de qualité de la cavité sera suffisant pour permettre l'oscillation. Nous empêchnns ainsi l'apparition du ou des premiers " pulses" qui étaient justement obtenus au moment où le parallélisme n'était pas atteint.
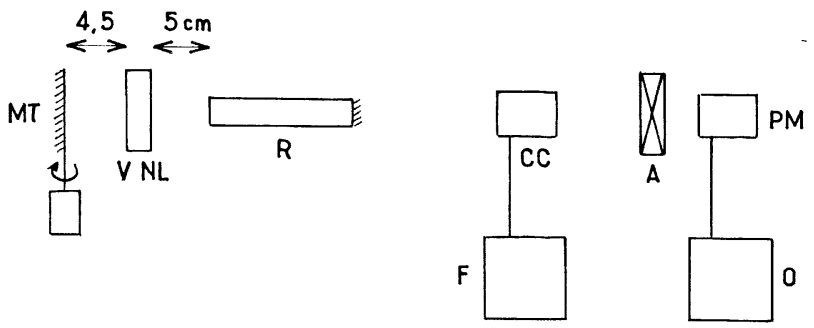

Fig. 5. - Schéma d'un laser à rubis déclenché par un miroir tournant associé à un verre non linéaire.

MT : miroir tournant; VNL : verre non linéaire Schott RG8 $2 \mathrm{~cm}$; $\mathrm{R}$ : rubis ; $\mathrm{CC}$ : calorimètre à cône ; F : fluxmètre ; A : atténuateur ; PM : photomultiplicateur $56 \mathrm{CVP}$; O : oscillographe Tektronix $585 \mathrm{~A}$.

Nous avons done réalisé le montage suivant : sur le banc d'optique nous plaçons successivement un miroir tournant (29 200 tours/mn) un verre Schott RG 8 et un rubis ; la face de sortie du rubis joue le rôle de miroir partiellement réfléchissant. Le pompage est assuré par un flash droit au xénon (fig. 5).

Nous avons réduit à $2 \mathrm{~mm}$ l'épaisseur des verres Schott précédemment employés afin que le montage permette d'atteindre le seuil d'oscillation.

Par cette méthode nous obtenons à l'oscillographe une impulsion unique. Sa largeur 11 nanosecondes et son énergie donnée par un calorimètre à cône étalonné nous permettent d'évaluer sa puissance à $45 \mathrm{MW}$ ( fig. 6).

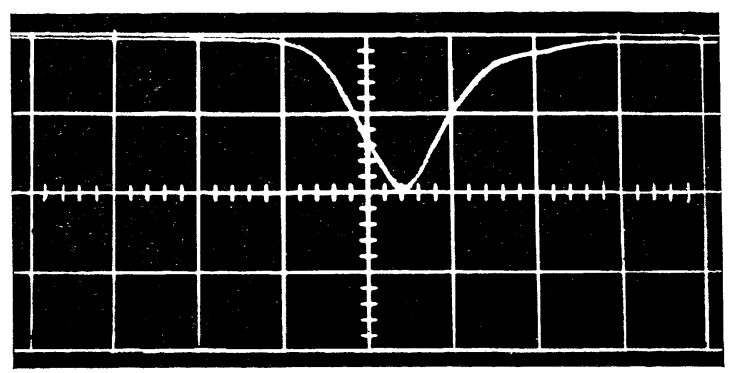

Fig. 6. - Oscillogramme d'un éclair de laser déclenché par un miroir tournant associé à un verre non linéaire. Sensibilité : $4 \mathrm{~V} / \mathrm{cm}$; balayage : $10 \mathrm{~ns} / \mathrm{cm}$. 
Nous constatons ici que :

- la puissance maximale est supérieure à celle obtenue dans le déclenchement à miroir tournant et dans le déclenchement à verre non linéaire;

- la largeur de l'impulsion est réduite de $30 \mathrm{~ns}$ à 1 i ns ;

- quelle que soit l'énergie du flash, au-dessus du seuil, nous n'obtenons qu'une impulsion (ce qui n'est pas le cas précédemment).

Conclusion. - L'association du miroir tournant au verre non linéaire nous a donc permis d'obtenir dans les conditions particulières de notre montage une impulsion unique, reproductible, plus puissante et plus courte.

Note : Nous apprenons que dans le livre du Dr Ross : "Laser licht verstärker und oscillation ", édité par Akademische Verlagsgesellschaff, Frankfurt-an-Main, qui doit paraître le 15 mars, le principe de cette méthode est donné.

Manuscrit reçu le 9 mars 1966

\section{BIBLIOGRAPHIE}

[1] Gires et Combaud, J. Physique, 1965, 26, 325.

[2] Bret et Grees, C. R. Acad. Sc. de Paris, 1964, 258, $4702-4705$. 